\title{
A importância do SLA no setor de telecomunicações
}

Adda R egina deO liveira Pimentel (UFF) - addapimentel@yahoo.com.br

H eitor L uiz M urat deM eirellesQuintella (UFF) - hquintel@unisys.com.br

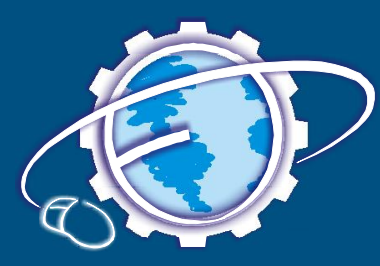

\section{Resumo}

No cenário atual de Telecomunicações, hoje a grande preocupação das empresas prestadoras de serviços é com a "qualidade percebida pelo cliente". A estratégia adotada pelos provedores de telecomunicações para atuar com sucesso sobre a qualidade percebida é a obtenção do Acordo de Nível de Serviço (SLA - Service Level Agreement) entre clientes e provedores. Este estudo teve por objetivos: comparar as percepções e expectativas dos clientes sobre a qualidade do SLA no serviço de comunicação de dados; analisar a quais aspectos do SLA os clientes e provedores de telecomunicações atribuem importância maior; e avaliar 0 atual estágio das ferramentas de TI em uso pelos provedores de telecomunicações para a gestão do SLA. Este trabalho tomou como base os modelos conceituais de Parasuraman et al. (1990) e a Cadeia de Valor Agregado desenvolvido por Porter (1985). Os resultados apontaram que o grau de satisfação dos clientes quanto ao SLA ofertado ainda não atende às suas expectativas, mas a partir desta pesquisa será possível fornecer subsídios aos provedores de telecomunicações para obtenção de melhorias em seus serviços, o que contribuirá para o sucesso do seu negócio, através do aumento da qualidade percebida por seus clientes.

Palavras-chave: SLA, qualidade percebida e tecnologia da informação.

\section{THE IMPORTANCE OF SLA IN TELECO MUNICATIONS INDUSTRY}

\begin{abstract}
In the current telecommunications scenario, one of the key points telecommunication service providers are focusing nowadays is the "customer perceived quality". The adopted strategy by those service providers to successfully act on the perceived quality in telecommunications services is the Service Level Agreement to be established between customers and suppliers. The present paper main objectives are the following: (i) to compare the customer's perceptions and expectations about SLA quality in the Data Communication Service; (ii) to analyze which SLA aspects both telecommunications customers and suppliers pay more attention and importance to; (iii) to analyze the IT tools currently in use by telecommunication providers for SLA management. This work is based on the models of Parasuraman et al. (1990), and the Value Added Chain developed by Porter (1985). The results show that the customers are not satisfied and that the offered SLA does not meet customer's expectations. In this way, based on the research presented herein and its related information, the telecommunications suppliers will be able to improve their services, taking the quality perceived for the customers as a key point for the success of their business.
\end{abstract}

Keywords: SLA, perceived quality and information tecnology. 


\section{INTRO DUÇÃO}

No cenário atual de Telecomunicações, a qualidade de serviço está no centro das atenções. Até a década de 1970, o foco das operações era voltado para a eficiência interna, tendo-se revertido, principalmente, após a privatização do setor de telecomunicações para o atendimento das demandas do mercado, e o foco no cliente passou a ser primordial. A grande preocupação das empresas prestadoras de serviço de telecomunicações é hoje a "qualidade percebida pelo cliente".

A estratégia adotada pelos provedores de telecomunicações para atuar com sucesso sobre a qualidade percebida é a obtenção do Acordo de Nível de Serviço (SLA - Service Level Agreement) entre clientes e provedores.

Pode-se dizer que a qualidade do serviço percebida pelo cliente tem dois componentes: a qualidade técnica e a qualidade da interação. A qualidade técnica depende, em geral, de fatores tecnológicos que podem ser controlados pelo provedor de telecomunicações, enquanto a qualidade da interação depende de um fator Fora de Controle, ou seja, a percepção do cliente. Parasuraman et al. (1990) argumentaram que a qualidade percebida pelo cliente é a comparação entre as expectativas que o cliente tinha sobre o serviço e as experiências que teve na execução do serviço.

Para melhorar a qualidade técnica do serviço, o provedor de telecomunicações pode atuar nos fatores que afetam a sua entrega, isto é, nos elementos que habilitam a sua prestação e naqueles que afetam a qualidade da interação. A tecnologia tem possibilitado o aprimoramento técnico dos serviços e da qualidade da interação. Atualmente estão disponíveis diversos métodos e ferramentas para a gestão da base tecnológica e da sua infra-estrutura operacional.
Resta saber como ajudar o cliente a estabelecer as suas expectativas, conciliando-as com o serviço real ofertado. Isso pode ser alcançado fazendo que o cliente tenha, no momento da contratação, uma clara compreensão da qualidade técnica e operacional que ele pode esperar.

A Gestão do Nível de Serviço ou gestão do SLA é o conjunto de processos e procedimentos que são aplicados para assegurar que o nível de serviço adequado seja prestado ao cliente. A adequação do nível de serviço deve ser definida de acordo com as prioridades das partes envolvidas, e dentro dos custos estabelecidos. O conceito de Gestão de Nível de Serviço foi desenvolvido para tornar disponível ao provedor as ferramentas de TI necessárias para gestão da qualidade do serviço e baseiase na definição e gestão de Acordos de Nível de Serviço (SLA - Service Level Agreement).

Segundo a recomendação E.860 da ITU-T (2002), o SLA é um acordo estabelecido entre o provedor e seu cliente para definir formalmente o nível de qualidade que deve ser atingido na prestação de um serviço. O objetivo é tornar a medição da qualidade objetiva e quantificável, introduzindo meios para mensurar a expectativa do cliente e, portanto, para gerir a qualidade percebida por ele. A gestão da qualidade percebida pelo cliente só é possível a partir do uso adequado de ferramentas de TI pelos provedores de telecomunicações.

Nesse contexto, os principais provedores de telecomunicações no Brasil, segundo o Atlas Brasileiro de Telecomunicações (2006) para a prestação de serviços de comunicação de dados ao mercado corporativo brasileiro, coincidem com as empresas concessionárias do Serviço Telefônico Fixo Comutado (STFC). A relação de provedores, bem como os serviços de comunicação de dados, está relacionada de acordo com a Tabela 1

Tabela 1 - Serviços corporativos dos provedores de telecomunicações

\begin{tabular}{|l|l|}
\hline Provedor de Telecomunicações & \multicolumn{1}{|c|}{ Tecnologias } \\
\hline Brasil Telecom & IP, MPLS, ATM, Frame-Relay, redes determinísticas, ethernet,x.25 \\
\hline CTBC Telecom & MPLS, Frame Relay, ATM, Ethernet, IP, ADSL, ISDN, GPRS, Edge \\
\hline Embratel & MPLS, Frame Relay, Satélite, ATM, X.25, redes determinpisticas, ADSL \\
\hline GVT & Frame Realy, ATM, ADSL \\
\hline Intelig & ATM, IP, Satélite \\
\hline Sercomtel & ATM, Frame Relay, rede determinística, ADSL \\
\hline Telemar & ADSL, satélite, Frame Relay, ATM, X.25, MPLS, IP, Metroethernet, redes determinísticas \\
\hline Telefônica Empresas & Frame Realy, ATM, ADSL, MPLS \\
\hline
\end{tabular}

Fonte: Atlas Brasileiro de Telecomunicações. 
Considerando-se esse cenário, este estudo teve como objetivos comparar as percepções e expectativas dos clientes sobre a qualidade do SLA e identificar o atual estágio das ferramentas de TI em uso pelos provedores de telecomunicações para a gestão do SLA. Este estudo traz como foco os serviços de comunicação de dados corporativos. .

\section{PRO BLEMA}

Assim, esta pesquisa tencionou avaliar: "O SLA dos serviços de comunicação de dados disponibilizados pelos provedores de telecomunicações estão satisfazendo as expectativas dos clientes?"

Este problema se insere na pesquisa Fatores $\mathrm{Hu}-$ manos e Tecnológicos da Competitividade conduzida na Universidade Federal Fluminense (QUINTELLA,1997).

\section{REFERENCIAL TEÓ RICO}

O referencial teórico utilizado no desenvolvimento deste estudo é composto pelo instrumento de avaliação da qualidade de serviços - SERVQUAL, desenvolvido por A.PARASURAMAN, Valarie A. ZEITHAML e Leonard L. BERRY para análise do Service Level Agreement (SLA) nos serviços de telecomunicações; e uso competitivo de tecnologia da informação para a Gestão do SLA, utilizando-se como referencial a Cadeia de Valor de Porter (1986).

\subsection{Q ualidade em Serviços}

Em 1983, A. PARASURAMAN, Valarie A. ZEITHAML e Leonard L. BERRY iniciaram um estudo sobre a qualidade do serviço, interessados em responder às seguintes questões:

O que é qualidade do serviço?

Quais são as causas dos problemas na qualidade dos serviços?

Que podem fazer as organizações para resolver esses problemas e melhorar seus serviços?

$O$ estudo consistiu em quatro fases:

A fase I consistiu em um estudo qualitativo do serviço aos clientes e aos executivos de empresas de serviços que, como resultado, permitiu desenvolver o modelo conceitual de qualidade do serviço.

A fase II consistiu num estudo empírico em grande escala que se centrou no ponto de vista do cliente no modelo de qualidade do serviço desenvolvido na fase I, desenvolvendo-se a partir desta fase uma metodologia para medir a qualidade do serviço, a "SERVQUAL".

A fase III consistiu num estudo empírico centrado na outra metade do modelo de qualidade do serviço, os fornecedores do serviço.

As fases I, II e III foram compostas de entrevistas, sessões de grupo com clientes, sessões de grupo para empregados, entrevistas em profundidade com executivos, estudos do consumidor, sondagens a diretores e empregados da linha de frente de atendimento ao público. Essas fases foram realizadas em seis áreas do setor de serviços: conserto e manutenção de equipamentos, cartões de crédito, companhias de seguros, ligações telefônicas de longa distância, serviços bancários e corretagem de valores.

A fase IV centrou-se nas expectativas que os clientes têm sobre o serviço, a forma como os usuários criam suas expectativas e os fatores-chave que afetam o processo. Incluem-se nesta fase outros serviços, que não haviam sido incluídos nas fases anteriores, como: serviços automotores, serviços de equipamento industrial, hotéis e aluguéis de caminhões.

Os resultados desta primeira fase apresentaram certos padrões nas respostas dos entrevistados que ajudaram a definir a qualidade do serviço, os fatores que influem as expectativas e os critérios de avaliação da qualidade do serviço, como mostrado na Figura 1.

Estudos estatísticos posteriores na estruturação do SERVQUAL mostraram uma importante correlação entre os critérios, concluindo que eles podem ser representados por cinco dimensões: Tangibilidade, Confiabilidade, Capacidade de Resposta, Segurança e Empatia.

Zeithaml et al. (1990) afirmaram que as cinco dimensões SERVQUAL conformam uma representação precisa das dimensões que os usuários utilizam para avaliar a qualidade dos serviços, já que constituem o resultado da análise sistemática das avaliações realizadas com centenas de entrevistados em vários setores do serviço. A seguir, uma breve descrição de cada uma delas.

Tangibilidade refere-se às instalações físicas, equipamentos, pessoal e material de comunicação.

Confiabilidade é a habilidade de se ter o que foi prometido, na data aprazada.

Capacidade de resposta é a habilidade de entender os problemas e dificuldades dos clientes e proporcionar o serviço prontamente.

Segurança é percepção que o cliente tem da habilidade do empregado da empresa em transmitir confiança, segurança e credibilidade.

E mpatia é a atenção individualizada, facilidade de acesso e comunicação que as empresas oferecem aos clientes. 


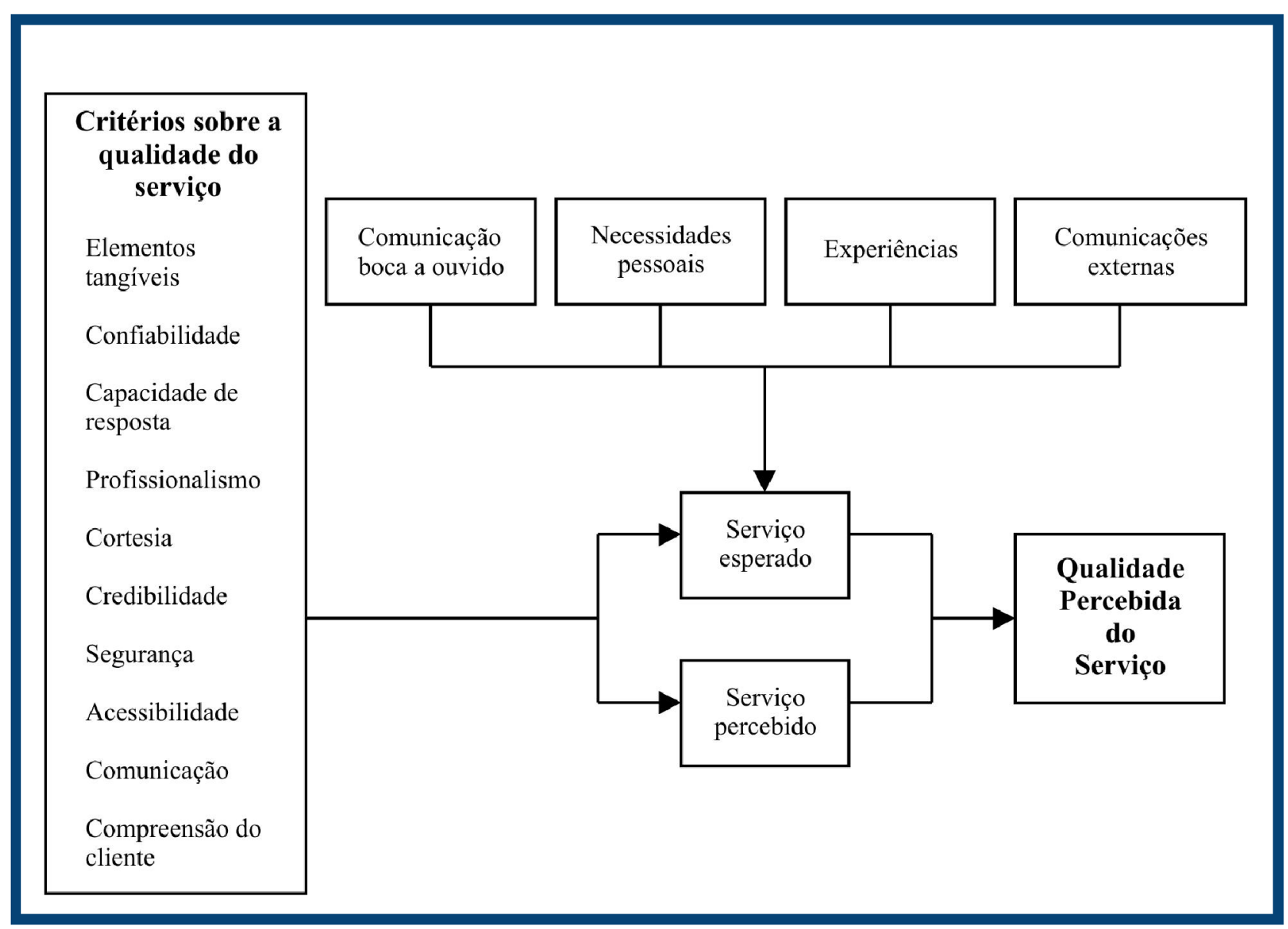

Figura 1 - Avaliação do cliente sobre a qualidade do serviço. Fonte: ZEITHAML et al., 1990.

\subsection{Modelo Conceitual da Qualidade do Serviço}

O modelo proposto por Parasuraman et al. (1985) vincula as discrepâncias que os clientes percebem na qualidade dos serviços - hiato 5 - com as discrepâncias internas que existem nas empresas fornecedoras de serviços. Portanto, esse hiato representa as discrepâncias potenciais que podem existir, do ponto de vista do cliente, entre o serviço esperado e o serviço percebido.

$\mathrm{O}$ resultado de seus estudos foi esquematizado em um modelo ampliado das discrepâncias da qualidade do serviço, em que se apresentam as deficiências da qualidade do serviço, suas causas e as dimensões que os clientes consideram na avaliação da qualidade do serviço.

Por ter sido desenvolvida em setores diversos, o modelo conceitual de Qualidade do Serviço pretende ser genérica e aplicável a todos os tipos de organização e ramos de serviços, se necessário com a introdução de adaptações, como sugerem os próprios autores.

\subsection{Aplicações}

Valarie A. Zeithalm, A. Parasuraman e Leonard L. Berry são pioneiros no estabelecimento do marketing de serviços como uma área diferente do marketing de produtos. Os referidos autores utilizaram como princípio para elaboração da pesquisa o modelo SERVQUAL, cujo principal objetivo é avaliar a qualidade dos serviços prestados por determinada empresa, e com base nos resultados da pesquisa as áreas consideradas fracas podem ser claramente identificadas e corrigidas. Além disso, esta pesquisa apontou, também, as áreas fortes da empresa, que podem ser usadas como vantagens competitivas.

Os resultados da pesquisa SERVQUAL proporcionaram insights importantes aos níveis gerenciais das 
empresas com respeito às medidas que deveriam ser tomadas para lograr um controle de qualidade eficaz nos serviços. Esses insights foram distribuídos em quatro classes de discrepâncias ou hiatos que se relacionam com as percepções que os executivos têm sobre a qualidade dos serviços e as funções associadas com a sua prestação aos clientes.

Com isso, o modelo SERVQUAL torna-se a importante ferramenta para garantir que a prestação de serviços esteja alinhada com a percepção de qualidade dos clientes e, mais ainda, que possa atender aos níveis de satisfação do mercado e, conseqüentemente, garantir a fidelização de seus clientes, bem como contribuir para a obtenção de novos clientes.

\subsection{A cadeia de valor}

Em 1983, Michael Porter introduziu o Modelo de Cadeia de Valor como uma ferramenta de avaliação de negócios, com base na posição estratégica de fornecedores, compradores e competidores existentes e futuros.

O modelo de Porter (1985) descreve que toda empresa é uma reunião de atividades que são executadas para projetar, produzir, comercializar, entregar e sustentar o produto ou serviço. Todas essas atividades podem ser representadas, fazendo-se uso de uma cadeia de valores.

Em termos competitivos, valor é o montante que os compradores estão dispostos a pagar por aquilo que uma empresa lhes fornece. As atividades de valor podem ser divididas em "primárias" e "de apoio" (PORTER, 1985).

As atividades primárias são aquelas envolvidas na criação física do produto ou serviço, na sua venda e transferência para o comprador, bem como na assistência após a venda (logística interna, operações, logística externa, marketing, vendas e serviço).

As atividades de apoio sustentam as atividades primárias e a si mesmas, fornecendo insumos adquiridos, tecnologia, recursos humanos e várias funções no âmbito da empresa.

As atividades de valor são, portanto, os blocos de construção da vantagem competitiva. O modo como cada atividade é executada, combinada com sua economia, determinará se uma empresa tem custo alto ou baixo em relação à concorrência. $\mathrm{O}$ modo como cada atividade de valor é executada também irá determinar sua contribuição para as necessidades do comprador. Na Figura 2 é apresentado o modelo gráfico da cadeia de valor proposto por Porter (1985).

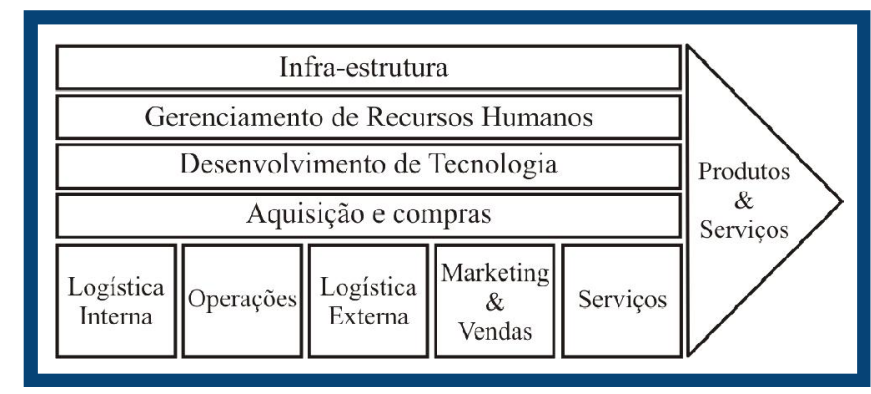

Figura 2 - Modelo de cadeia de valor. Fonte: PORTER, 1985.

\subsection{Aplicações}

Nos últimos 20 anos, muitas empresas de consultoria em negócios e diversas pesquisas científicas usaram esse modelo para:

I Identificar as atividades desenvolvidas em cada unidade de negócios.

I Analisar o valor criado nessas unidades de negócio.

I Examinar os fluxos de entrada e saída em cada unidade.

I Mapear as trocas de entrada e saída de uma organização.

I Identificar atividades críticas para o sucesso.

I Entender a alocação de recursos e a sua correspondência com as contribuições de valor de determinada atividade.

Embora esse modelo tenha sido publicado há 20 anos, ele continua sendo largamente aplicado na análise de competitividade entre empresas e setores da economia.

\section{HIPÓTESES DA PESQUISA}

As hipóteses em estudo foram: (a) Hipótese 1: existem diferenças entre as percepções e expectativas que os clientes das empresas de telecomunicações têm da qualidade do SLA dos serviços de comunicação de dados; (b) Hipótese 2: existem diferenças entre o ponto de vista das empresas de telecomunicações e o de seus clientes quanto à importância relativa de cada uma das dimensões de avaliação da qualidade do SLA dos serviços de comunicação de dados; (c) Hipótese 3: as empresas de telecomunicações dispõem de ferramentas de TI adequadas para a gestão do Nível de Serviço no provimento de serviços de comunicação de dados. 


\section{METO DO LO GIA}

O método de abordagem utilizado para a realização da pesquisa foi o Hipotético-Dedutivo de Popper (POPPER, 1993), conforme referência.

Marconi e Lakatos (2003, p. 83), conforme referência, definiram o método científico como o conjunto das atividades sistemáticas e racionais que, com maior segurança e economia, permitem alcançar o objetivo conhecimentos válidos e verdadeiros -, traçando o caminho a ser seguido, detectando erros e auxiliando as decisões do cientista.

O método científico empregado nesta pesquisa foi o método hipotético-dedutivo. Este método propõe que o único conhecimento genuíno é o obtido pelos caminhos da ciência. As únicas verdades seguras são as que se podem avaliar com objetividade, independentemente do observador. Deduz resultados a partir da formulação de hipóteses. Submete as hipóteses a testes e busca regularidade e relacionamentos causais entre seus elementos. Seu critério original, a verificabilidade, toma por base o princípio de que a proposição que não for passível de comprovação científica não faz sentido. Pelo critério da falseabilidade, proposto por Karl Popper, são as tentativas sem êxito de falsear uma proposição que lhe dão ainda mais validade (JAPIASSÚ; MARCONDES, 1996, p. 217).

\subsection{Classificação quanto ao Tipo de Pesquisa}

Quanto às técnicas de pesquisa empregadas, de acordo com Marconi e Lakatos (2003), esta pesquisa pode ser classificada como uma pesquisa de campo, quantitativo-descritiva. Uma pesquisa de campo é aquela utilizada com o objetivo de conseguir informações e, ou, conhecimentos acerca de um problema para o qual se procura uma resposta, ou de uma hipótese que se queira comprovar ou, ainda, descobrir novos fenômenos ou as relações entre eles. O que principalmente caracteriza esta pesquisa como de campo é o levantamento no mercado (campo) das percepções dos clientes corporativos e provedores de telecomunicações sobre o SLA associado ao serviço de comunicação de dados.

Um dos tipos de pesquisa de campo é a pesquisa quantitativo-descritiva. Para Marconi e Lakatos (2003), a pesquisa de campo quantitativo-descritiva consiste em investigações empíricas, que objetivam o delineamento ou análise das características principais ou decisivas de um fenômeno, a avaliação de programas ou, ainda, o isolamento de variáveis principais ou chave.

Neste tipo de estudo são empregadas técnicas como entrevistas e questionários e procedimentos de amostragem.

\subsection{Alvos da Pesquisa}

O público-alvo da pesquisa foi constituído por dois grupos distintos:

Gerentes de operações dos provedores de telecomunicações no Brasil que fornecem serviços de comunicação de dados.

Gerentes responsáveis pelas áreas de telecomunicações/TI de empresas de grande porte, as quais são clientes de serviços de comunicação de dados.

Para cada grupo (população) foi extraída uma amostra. Segundo Vergara (1998), uma amostra é uma parte da população, escolhida segundo algum critério de representatividade. As amostras são assim caracterizadas:

a) A mostra de provedores de telecomunicações:

Participaram da pesquisa 10 respondentes de cinco provedores de telecomunicações que oferecem serviços de comunicação de dados nas diversas regiões do Brasil, a saber: Brasil Telecom, Embratel, Intelig, Telefônica e Telemar. Para a amostra de provedores, o julgamento foi fator-chave na seleção dos respondentes. Julgou-se que os gerentes e profissionais da área de operações eram os mais indicados para responder à pesquisa, por serem os responsáveis pelo gerenciamento das plataformas de serviços de comunicação de dados e pelo desenvolvimento de estratégias de lançamento de serviços de gerência voltados para clientes.

A amostra de provedores de telecomunicações foi bastante representativa da população, pois quase a totalidade dos provedores participaram da pesquisa. Apenas os provedores Sercomtel, CTBC Telecom e GVT não participaram da pesquisa. Esses provedores juntos detêm participação de mercado inferior a $20 \%$.

b) A mostra de clientes:

Trinta clientes (30) corporativos de serviços de comunicação de dados participaram da pesquisa. Para os clientes de serviços de comunicação de dados, a amostragem foi realizada por conveniência. O questionário foi disponibilizado na Internet, e através da distribuição do link para alguns contatos ele foi sendo divulgado a outros clientes. Esse tipo de amostragem foi escolhido devido a limitações na realização de outro tipo de amostragem, como dispêndio de recursos e tempo necessário à sua realização. 


\subsection{Coleta de Dados}

$\mathrm{O}$ instrumento de coleta de dados utilizado nesta pesquisa foi o questionário autopreenchido. O questionário desenvolvido pode também ser classificado como medianamente estruturado e não disfarçado. Os pesquisados responderam a perguntas fixas e tiveram total conhecimento sobre os propósitos da pesquisa. $\mathrm{O}$ último bloco do questionário foi destinado a respostas livres e de caráter opcional. Em razão disso, foram obtidos alguns insights importantes sobre fatores de qualidade do SLA, de acordo com a percepção dos respondentes.

\subsection{Tratamento e Análise dos Dados}

A partir da análise dos instrumentos de coleta de dados e dos métodos de avaliação destes (MATTAR, 1997) foram desenvolvidos dois modelos de questionário. Cada questionário destinou-se a uma amostra específica - clientes e provedores de telecomunicações, na qual foi possível recolher todos os dados necessários ao desenvolvimento deste trabalho.

Segundo Mattar (1997), a escolha do método estatístico depende de fatores como a escala de mensuração utilizada e o grau de relacionamento entre as amostras. Com base nessas considerações, os métodos escolhidos para o tratamento dos dados foram o método t-Student e o qui-quadrado, além do uso da Lógica Paraconsistente.

\section{ANÁLISE DE RESULTADOS}

\section{Hipótese I}

A Hipótese I está relacionada ao Hiato 5 do modelo conceitual de qualidade em serviços de Parasuramn et al. (1985). Foram utilizadas neste trabalho três dimensões do modelo SERVQUAL, a "Tangibilidade", a "Confiabilidade" e a "Capacidade de Resposta". A opção por tal sistemática se deve ao fato de o trabalho ser relacionado à qualidade de SLA, em que é estabelecido um acordo envolvendo essas três dimensões. Daí o motivo pela opção e a convicção de que as três dimensões são as mais apropriadas para o referido trabalho. As avaliações apresentadas ao final deste artigo comprovam tal afirmativa.

Para fins desta pesquisa foi levado em conta o "Gap 5" do modelo de Parasuraman et al. (1990), ou seja, a diferença total da percepção e da expectativa da qualidade do SLA dos serviços de comunicação de dados prestados pelos provedores de telecomunicações. Os dados foram tratados estatisticamente pela distribuição $t$ (distribuição de Student), para pequenas amostras (n ? 30), mas, de acordo com Anderson et al. (2003), ela não se restringe às pequenas amostras, podendo ser utilizada com o intuito de corroborar ou refutar as hipóteses de teste da diferença entre duas médias da população, para comprovar, ou não, a existência do "gap". Os dados amostrados são exibidos na Tabela 2.

Tabela 2 - Dados de análise

\begin{tabular}{|l|c|c|c|c|}
\hline \multicolumn{1}{|c|}{ Dimensão } & Percepção & Expectativa & Gap 5 & Importância \\
\hline Tangibilidade & 5,86 & 7,97 & $-2,11$ & $21,27 \%$ \\
\hline Confiabilidade & 5,83 & 7,98 & $-2,16$ & $41,43 \%$ \\
\hline $\begin{array}{l}\text { Capacidade de } \\
\text { Resposta }\end{array}$ & 5,28 & 7,96 & $-2,68$ & $37,30 \%$ \\
\hline
\end{tabular}

Fonte: Elaboração própria.

Conforme observado na Tabela 1 , as diferenças entre percepção e expectativa, que caracterizam o Hiato 5, são consideradas significativas nas três dimensões em estudo. Desse modo, a hipótese de nulidade de que as médias das percepções são equivalentes às médias das expectativas, com 95\% de confiança, pode ser refutada.

Pode-se observar, no gráfico da Figura 3, a "tangibilidade" como a dimensão com a menor distância da expectativa dos clientes entrevistados, tendo ela o menor hiato (gap) e a "capacidade de resposta" à dimensão de maior diferença entre a percepção e a expectativa da qualidade do SLA.

Com relação à importância dada para cada dimensão anterior, os dados se apresentam como na Figura 4.

Observa-se que a dimensão "confiabilidade" foi a que teve a maior importância para os clientes entrevistados, sendo seguida pela "capacidade de resposta".

Foi analisada ainda, para a Hipótese I, a lógica Paraconsistente, que na prática funciona da seguinte forma: 1) Se existir alto grau de contradição, não haverá certeza ainda quanto à decisão, portanto se devem buscar novas evidências.

2) Se existir um baixo grau de contradição, poder-se-á formular a conclusão, desde que se tenha alto grau de certeza.

A análise paraconsistente dos graus de crença e descrença pode ser feita através da representação do reticulado em um Quadrado Unitário no Plano Cartesiano - QUPC, em que os graus de crença ficam no eixo $\mathrm{X}$ e os de descrença, no eixo Y. Veja a representação dos resultados na Figura 5. 


\section{Dimensões Servqual para SLA}

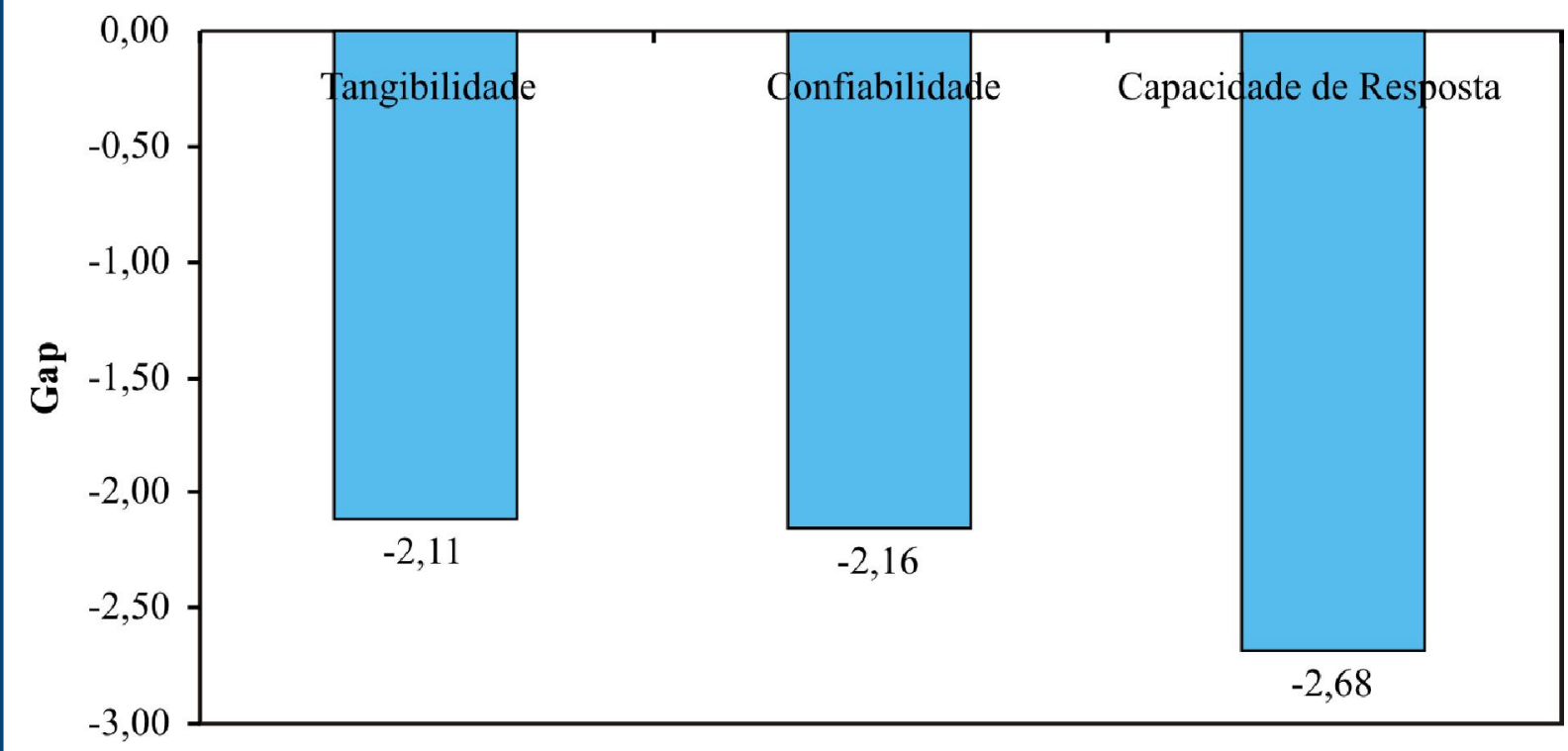

Figura 3 - Dimensões Servqual do SLA.

Fonte: Elaboração própria.

\section{Importância das Dimensões Servqual para SLA}

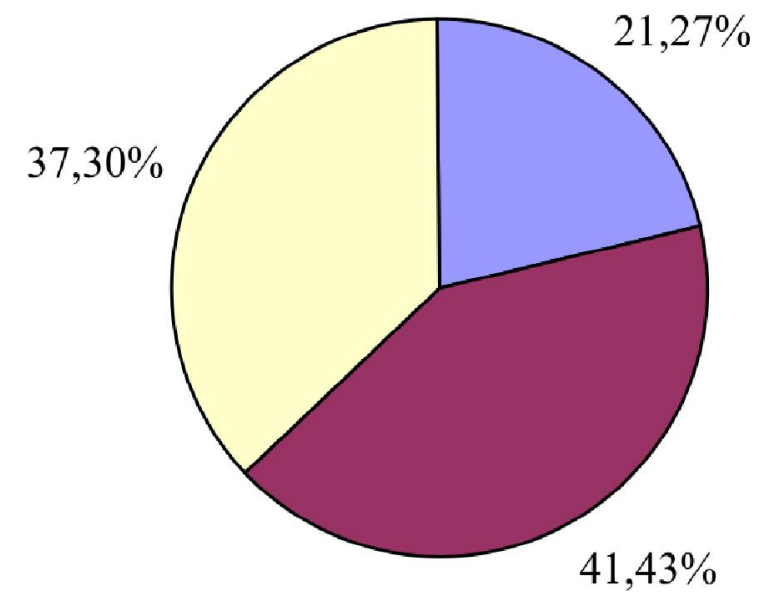

$\square$ Tangibilidade

$\square$ Confiabilidade

$\square$ Capacidade de Resposta

Figura 4 - Importância das Dimensões Servqual do SLA.

Fonte: Elaboração própria. 
No Quadrado Unitário no Plano Cartesiano, têm-se as seguintes notações:

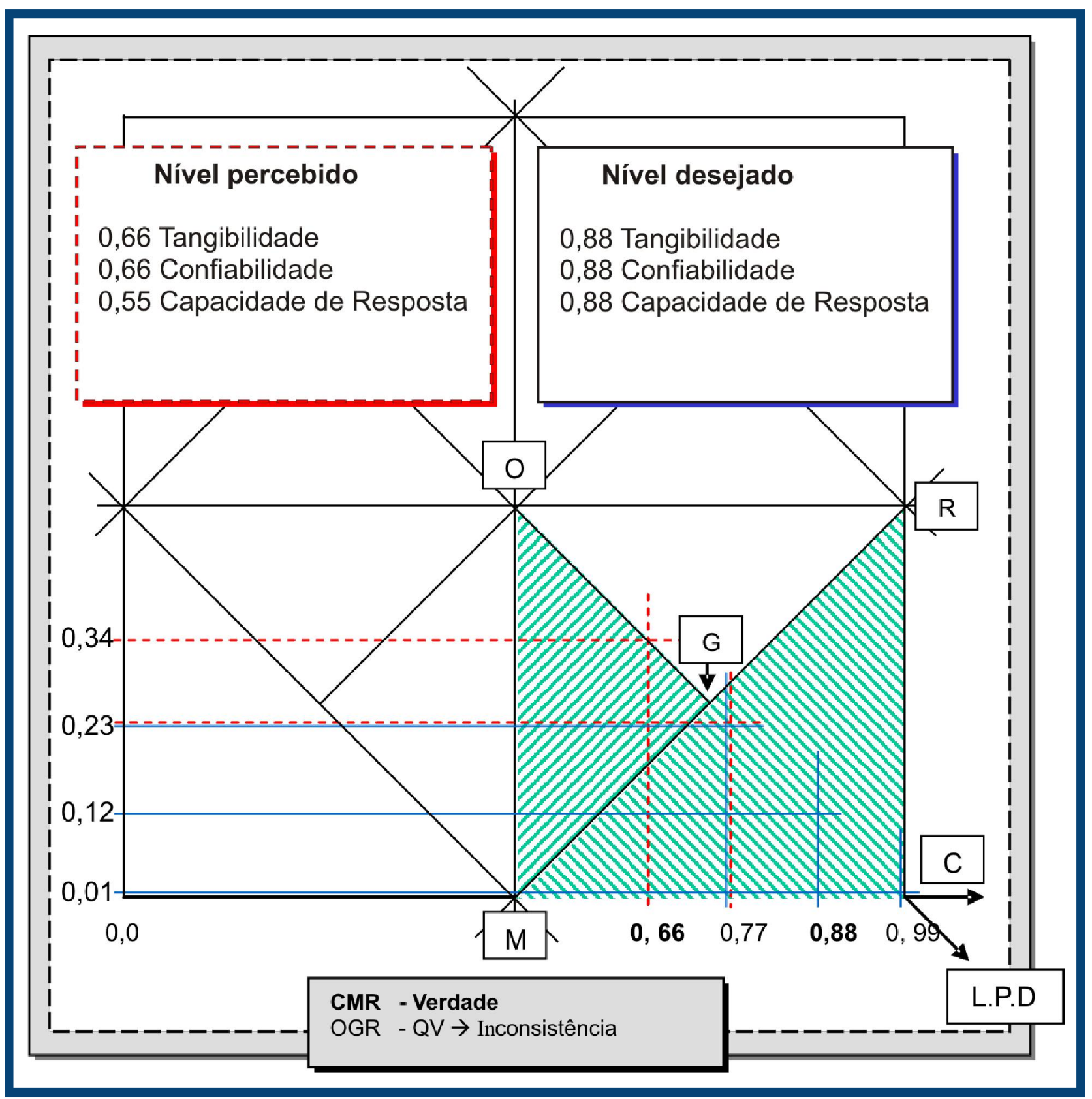

Figura 5 - Resultado do emprego da comparação entre o nível percebido e o nível desejado.

Fonte: Elaboração própria.

Com base no resultado do nível percebido e nível desejado representado no Quadrado Unitário no Plano Cartesiano, verifica-se que:

I Nível desejado:

Os aspectos Tangibilidade, Confiabilidade e Capacidade de Resposta estão na região CMR e correspondem à VERDADE. Assim, pode-se concluir que as aspirações dos clientes são viáveis.
I Nível percebido:

Os aspectos Tangibilidade e Confiabilidade estão na região OGR e correspondem à QUASE-VERDADE, tendendo a INCONSISTENTE. Assim, pode-se concluir que existe inconsistência nas aspirações dos clientes.

O aspecto Capacidade de Resposta está na região OGM e corresponde à QUASE-VERDADE, com 
tendência a INDETERMINADO, podendo-se concluir que existe indeterminação nas aspirações dos clientes.

De acordo com os resultados da Hipótese I, é possível afirmar que existem diferenças entre as percepções e expectativas que os clientes têm da qualidade do SLA dos serviços de comunicação de dados.

\section{Hipótese II}

A Hipótese II está relacionada aos Hiatos 1 e 5 do modelo conceitual de qualidade em serviços de Parasuraman et al. (1985). Para avaliação da importância relativa das dimensões da qualidade do SLA, os dados foram tratados estatisticamente pela distribuição qui-quadrado. Os dados amostrados são exibidos na Tabela 3.

Tabela 3 - Tabulação dos dados referentes à hipótese II

\begin{tabular}{|l|c|c|c|}
\hline \multicolumn{1}{|c|}{ Dimensão } & $\begin{array}{c}\text { Ponto de Vista } \\
\text { dos Provedores }\end{array}$ & $\begin{array}{c}\text { Ponto de vista } \\
\text { dos clientes }\end{array}$ & Difer ença \\
\hline Tangibilidade & 22,50 & 21,27 & 1,23 \\
\hline Confiabilidade & 41,50 & 41,43 & 0,07 \\
\hline $\begin{array}{l}\text { Capacidade de } \\
\text { Resposta }\end{array}$ & 36,00 & 37,30 & $-1,30$ \\
\hline
\end{tabular}

Fonte: Elaboração própria.

Conforme pode ser observado na Tabela 2, as diferenças entre os pontos de vista de clientes e provedores de telecomunicações não pareceram significativas em todas as dimensões estudadas. Portanto, a hipótese de nulidade de que as médias dos pontos de vista de clientes e provedores de telecomunicações são equivalentes, com 95\% de confiança, pode ser refutada.

\section{Hipótese III}

A avaliação dos sistemas de suporte e das ferramentas de tecnologia de informação pelos profissionais das empresas de telecomunicações teve como objetivo avaliar a adequação às necessidades de gestão do SLA no ambiente atual do Setor de Telecomunicações. A avaliação teve como foco a investigação da importância e atendimento das atividades da Área Operacional, no que tange à gestão do SLA para prestar serviços de telecomunicações. A escolha pela área de operações se deveu ao fato de que as atividades de gestão do SLA no provimento de serviço de comunicação de dados são àquelas destinadas ao gerenciamento dos serviços sob a ótica do cliente, capacitando-o a monitorar o desempenho de sua rede de dados, os possíveis pontos de falha, agregando valor para ao seu próprio negócio e contribuindo pata atingir seus objetivos e metas.
Os participantes da pesquisa analisavam uma lista de atividades operacionais pertinentes à gestão do SLA e informavam se era importante ter sistemas de suporte e, em caso positivo, atribuíam um valor para o atendimento de cada atividade relacionada à Gestão do SLA.

A etapa da cadeia de valor descrita pelas atividades operacionais obteve índice de importância de 100\%, ou seja, os sistemas de suporte para a gestão do SLA têm grau de importância máximo percebido pelos profissionais do setor (Figura 4).

Tabela 4 - Resultado da avaliação de importância dos sistemas de suporte para gestão do SLA nas atividades de operações

\begin{tabular}{|c|c|c|}
\hline \multicolumn{3}{|c|}{ A valiação da importância de sistemas de suporte para as } \\
atividades de Oper ações \\
\hline $\begin{array}{c}\text { Quantidade de } \\
\text { Respostas } \\
\text { "Considera } \\
\text { importante ter } \\
\text { sistemas de } \\
\text { suporte" }\end{array}$ & $\begin{array}{c}\text { Quantidade de } \\
\text { Respostas "Não } \\
\text { considera } \\
\text { importante ter } \\
\text { sistemas de } \\
\text { suporte" }\end{array}$ & $\begin{array}{c}\text { Índice de } \\
\text { importância dos } \\
\text { sistemas de suporte } \\
\text { para atividade da } \\
\text { cadeia de valor }\end{array}$ \\
\hline 10 & 0 & $100 \%$ \\
\hline
\end{tabular}

Fonte: Elaboração própria.

A nota média dos sistemas de suporte para atendimento das atividades de Gestão do SLA foi menor que 76 pontos (limite inferior para um índice de confiança de $95 \%$ ), ou seja, a avaliação dos profissionais do setor leva à conclusão de que os sistemas de suporte ainda não se encontram num patamar razoável para garantir o cumprimento do SLA de forma eficaz e com agilidade, capaz de melhorar a percepção dos clientes corporativos que demandam cada vez mais um estabelecimento de contato de SLA com seus provedores.

Tabela 5 - Resultado da avaliação do atendimento dos sistemas de suporte para gestão do SLA nas atividades de operações

\begin{tabular}{|c|c|c|}
\hline \multicolumn{2}{|c|}{ Grau de atendimento de TI aos requisitos de G estão do SLA } \\
\hline Média & $\begin{array}{c}\text { Limite Superior } \\
\text { (ICM 95\%) }\end{array}$ & $\begin{array}{c}\text { Limite Inferior } \\
\text { (ICM 95\%) }\end{array}$ \\
\hline 73 & 76 & 64 \\
\hline
\end{tabular}

Fonte: Elaboração própria.

Comparando a importância e desempenho da TI na área operacional, verificou-se que os sistemas de suporte estão razoavelmente adequados aos requisitos de gestão do SLA (Figura 6). 


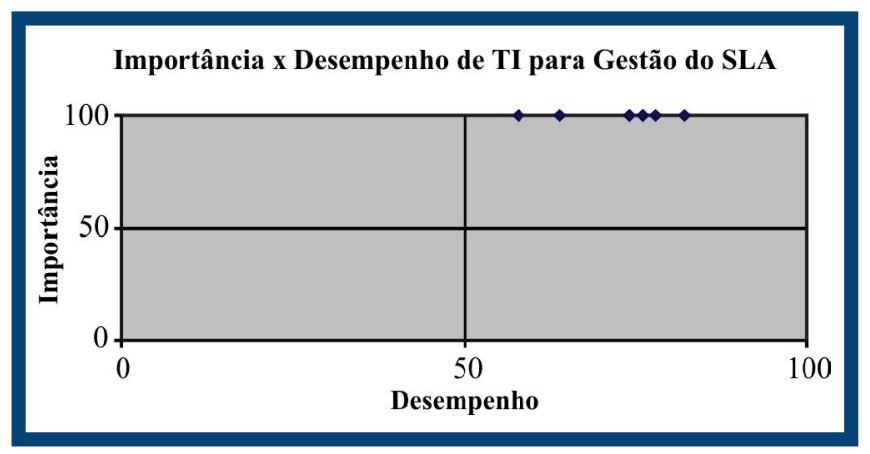

Figura 6 - Comparação entre desempenho e importância de TI.

Fonte: Elaboração própria.

De acordo com os resultados da Hipótese III, podese considerar que os provedores de telecomunicações precisam adequar suas ferramentas de TI, a fim de implementar a gestão do nível de serviço no provimento de serviços de comunicação de dados.

\section{CONCLUSÕES}

Depois da verificação de cada hipótese e da resposta às questões-chave, pôde ser feita uma análise dos resultados com relação à contextualização do problema e, a partir dessa análise, fazer inferências sobre as percepções respondentes, ou seja, do segmento em estudo. Pode-se, a partir dos resultados, concluir que:

As discrepâncias entre as percepções e as expectativas que os clientes corporativos têm da qualidade do SLA associado ao serviço de comunicação de dados prestados pelos provedores de telecomunicações são consideradas significativas nas três dimensões em estudo.

Apesar do pequeno tamanho da amostra e de acordo com a percepção da autora, pode-se concluir que existe um gap entre as percepções e expectativas do SLA ofertado. Essa afirmativa responde à principal interrogativa desta pesquisa, pois traz indícios de que os provedores de telecomunicações ainda não estão satisfazendo a qualidade do SLA desejado pelos clientes.

Para o provedor de telecomunicações, a qualidade do SLA em um serviço de comunicação de dados depende de um conjunto de procedimentos colocados em prática para garantir o cumprimento dos SLAs. A pesquisa em campo investigou tais fatores e, com base nos resultados, foi possível verificar que as expectativas dos clientes ainda não estão sendo atendidas.

Foram investigadas também as possíveis discrepâncias entre o ponto de vista dos provedores de teleco- municações e o de seus clientes, quanto à importância relativa de cada uma das dimensões de avaliação da qualidade do SLA.

De acordo com os resultados, não é possível afirmar que existem diferenças entre o ponto de vista das empresas de telecomunicações e o de seus clientes quanto à importância relativa das dimensões de avaliação da qualidade do SLA dos serviços de comunicação de dados.

Há, entretanto, grande probabilidade de esse fato estar ligado ao pequeno tamanho da amostra e à larga área de não-rejeição associada. Excluindo essa possibilidade, no entanto, pode-se especular que os provedores de telecomunicações pesquisados já tenham percebido a importância das dimensões relevantes para o SLA, apesar de ainda não ter implementado o uso de ferramentas adequadas conforme os resultados da hipótese 3 , que será explicada mais adiante.

Foi observado que tanto os provedores de telecomunicações quanto seus clientes deram maior importância à confiabilidade, vital para o cumprimento do SLA dos serviços de comunicação de dados oferecidos. Uma possível explicação poderia ser que determinadas características do serviço, consideradas fundamentais, não tenham recebido pontuação expressiva, pelo fato de a sua importância ser considerada óbvia pelos clientes, que concentrariam pontos em outras questões não muito bem trabalhadas pelos provedores de telecomunicações.

Adicionalmente, foi investigado nesta pesquisa se os provedores de telecomunicações dispõem de ferramentas de TI adequadas para a gestão do SLA no que tange às atividades operacionais pertinentes para essa gestão.

Como a quantidade de informação tratada na gestão de SLAs é enorme, faz-se necessário o apoio de uma ferramenta informatizada. Daí a necessidade de se investigar qual é o atual estágio das ferramentas de TI dos provedores de telecomunicações quanto à gestão do SLA.

Apesar de o grau de atendimento x importância das dimensões do SLA dado pelos provedores e clientes entrevistados ser bastante semelhante, os provedores ainda não dispõem de ferramentas de TI adequadas para a gestão do SLA. Talvez isso possa explicar o não- atingimento do nível de satisfação dos clientes em função da existência do gap 5.

Outro aspecto da análise é que muitos clientes desejam obter SLAs para seus serviços de comunicação de dados semelhantes aos que possuem em seus sistemas internos de TI, ou mesmo outros aspectos relativos ao nível de satisfação dos clientes como preço e segu- 
rança no atendimento podem, de alguma forma, ter influenciado o grau de satisfação dos clientes, mesmo com a indicação de que a pesquisa não tem o objetivo de analisar tais aspectos.

\section{SUGESTÕES PARA ESTUDOS FUTUROS}

A pesquisa sobre SLA não acaba neste trabalho. Outros aspectos originados a partir desta pesquisa também podem ser investigados mais aprofundadamente. A seguir, algumas sugestões para futuros estudos, que podem complementar e aprofundar este trabalho:

I Estender o estudo do SLA aos demais serviços de telecomunicações, bem como os demais aspectos de satisfação dos clientes.

I Ampliar amostra de clientes, pois foi pequena e retratou os provedores de primeira linha do Brasil e somente os clientes de grande porte do país.

1 Pesquisar os outros hiatos do modelo de Parasuraman et al. (1988), para permitir uma visão geral do processo de prestação do serviço. São eles: as discrepâncias entre as expectativas dos usuários e as percepções da gerência (Hiato 1); as discrepâncias entre o que a empresa entende como expectativa do cliente e a especificação do serviço (Hiato 2); as discrepâncias entre as especificações do serviço e o serviço realizado (Hiato 3); e as discrepâncias entre a comunicação aos clientes relativa ao serviço e o serviço realizado (Hiato 4).

I Investigar mais detalhadamente a implementação do Service Level Management -SLM nas outras atividades da cadeia de valor dos provedores de telecomunicações.

\section{REFERÊNCIAS}

\section{ATLAS BRASILEIRO DE TELECOMUNICAÇÕES. R evista} Teletime, 2006.

ITU-T - Telecommunication standardization sector of ITU. Recomen dação E .860. [S.1.]: Framework of a Service Level Agreement, 2002.

TELEMANAGEMENT FORUM. SL A management handbook. Vols. 1, 2 e 3. Disponível em: 〈http://www.tmforum.org>.

JAPIASSÚ, H.; MARCONDES, D. Dicionário básico de filosofia. Rio de Janeiro: Zahar, 1996.
LAKATOS, E. M.; MARCONI, M.A. M etodologia científica. 2. ed. São Paulo: Atlas, 1991.

LAKATOS, E. M.; MARCONI, M.A. Fundamentos da metodologia científica. São Paulo: Atlas, 1993.

LAKATOS, E. M.; MARCONI, M.A. M etodologia do trabalho científico. 5. ed. São Paulo: Atlas, 2001.

MATTAR, Najib Fauze. Pesquisa de marketing: metodologia, planejamento, execução, análise. 2. ed. São Paulo: Atlas, 1994. v. 1.

NETO, Maurício Correia Lemes; VENSON, Nério. Lógica paraconsistente. Florianópolis: UFSC, 2002.

PARASURAMAN, A.; ZEITHAML, Valarie A.; BERRY, Leonard L. A conceptual model of service quality and its implicans for future research. [S.1.]: Journal of Marketing, Fall, 1985.

PARASURAMAN, A.; ZEITHAML, Valarie A.; BERRY, Leonard L. Delivering quality service. New York: The Free Press, 1990.

POPPER, Karl R. Epistemologia e liberalismo, uma introdução à filosofia. Porto Alegre: Editora PUC/RS, 1993.

PORTER, Michael E. Vantagem competitiva. Rio de Janeiro: Editora Campus, 1986.

QUINTELLA, Heitor M. Fatores humanos e tecnológicos da competitividade. Niterói, RJ: Universidade Federal Fluminense, 1997. (Projeto de Pesquisa - Departamento de Engenharia Civil).

QUINTELLA, Heitor M. Um método de or ientação de mestrandos: relatório interno. Niterói, RJ: Universidade Federal Fluminense, 1999. (Apostila).

RUSSEL, Bertrand. Introdução à fil osofia da M atemática. Portugal: Livraria Almedina, 2001.

SIEGEL, S. Estatística não-paramétrica para as Ciências do Comportamento. São Paulo: McGraw-Hill do Brasil, 1981.

VERGARA, Sylvia Constant. Métodos de pesquisa em administração. São Paulo: Editora Atlas, 2005.

ZEITHAML, V. A.; PARASURAMAN, A.; BERRY, L. L. Delivering quality service. New York: The Free Press, 1990. 